\section{Schlechte Bakterien stören Wundheilung}

Durch eine Darmoperation kann sich die Zusammensetzung der Darmbakterien so verändern, dass sie giftige Substanzen freisetzen und die Wundheilung stören. Das haben Wissenschaftler um Prof. Dr. John Alverdy von der Universität Chicago im Tiermodell gezeigt. Das Ziel muss laut Alverdy sein, die "guten“ Bakterien im Darm zu fördern und die "schlechten“ zu verdrängen. Die Wissenschaftler forschen nun an einem Wirkstoff, der anders als Antibiotika keine Resistenzen fördert. Sie hoffen, so den Antibiotika-Einsatz zu reduzieren und multiresistente Krankenhauskeime in den Griff zu bekommen.

(eb)

Sci Trans/ Med. 2015;7:286ra68

\section{Reizdarm: Liegt's am Gluten?}

In der Studie GLUTOX wurden 140 Patienten mit unklaren gastrointestinalen Symptomen mittels einer doppelblinden, placebokontrollierten Glutenbelastung auf eine Gluten-/Weizensensitivität (Non-Celiac Gluten Sensitivity, NCGS) untersucht. Die Studie ergab, dass bei glutenfreier Ernährung drei von fünf Patienten nicht mehr an den Beschwerden litten, die zuvor auf ein Reizdarmsyndrom zurückgeführt wurden. Darüber hinaus reagierte einer von fünf Patienten mit Reizdarmsyndrom mit einer Verschlechterung der Symptome auf die blinde Wiedereinführung von Gluten. (eb)

Nutrients. 2016:8:84

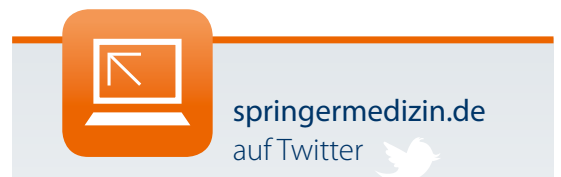

Im Frühling heißt es wieder: Lauschen Sie unserem Zwitschern und werden Sie zum Follower! twitter.com/springermedizin

Chronische Rhinosinusitis

\title{
Steroide direkt in Polypen spritzen!
}

Neben der topischen Steroidtherapie der Polyposis nasi könnte sich eine neue Methode etablieren: die Injektion relativ hoher Dosen von Kortikosteroiden direkt in die Polypen.

HNO-Ärzte aus Ankara haben für eine Studie 90 Patienten mit Nasenpolypen behandelt. Eine Gruppe erhielt zwei Wochen lang orales Prednisolon. Die initiale Tagesdosis von $1 \mathrm{mg} / \mathrm{kg}$ Körpergewicht wurde jeden Tag um $5 \mathrm{mg}$ reduziert. Die andere Gruppe bekam wöchentlich eine Injektion von $40 \mathrm{mg}$ Triamcinolon über fünf Wochen, gleichmäßig in die Polypen verteilt. Dann erhielten alle Patienten eine zwölfwöchige topische Therapie mit Fluticason-Nasentropfen. Vor der Therapie hatten alle $\mathrm{Pa}$ tienten im nasalen Symptomen- wie im Po- lypenscore Werte im Bereich mäßiger bis schwerer Ausprägung aufgewiesen. Bei den Kontrolluntersuchungen nach drei und sechs Monaten hatten sich die Scores in beiden Gruppen halbiert. Es zeigte sich kein signifikanter Unterschied zwischen der oralen Therapie und den Injektionen. Gleiches galt für die Auswertung der computertomografischen Aufnahmen der Nasennebenhöhlen.

Die gemessenen Kortisol- und ACTHSpiegel blieben in der Injektionsgruppe stets im Normbereich. Es traten keine schweren Komplikationen auf. Zu beachten ist, dass die intranasale Kortikoidinjektion zur Polyposis-Behandlung kein zugelassenes Verfahren ist.

Laryngoscope 2016, online 24. März

Keine TSH-Kosmetik!

\section{Hypothyreose nur bei Symptomen behandeln}

Erhöhte TSH-Spiegel bei normalen peripheren Schilddrüsenhormonspiegeln können rasch zum Rezeptblock greifen lassen. Für viele Patienten muss das kritisch hinterfragt werden.

Patienten mit subklinischer Hypothyreose unter 65 Jahren und einem TSHWert unter $10 \mathrm{mU} / 1$ sollten gemäß den Leitlinien nur dann behandelt werden, wenn auch Symptome einer Hypothyreose vorhanden sind.

Bei einem Wert von über $10 \mathrm{mU} / \mathrm{l}$ ist immer eine Behandlung indiziert. Bei $\mathrm{Pa}-$ tienten über 65 Jahren ist nach Angaben von Prof. Dr. Georg Brabant, Lübeck, aber Vorsicht geboten, da eine Therapie dann auch ungünstige Effekte haben kann.

Sinkende Grenzwerte und steigende Schilddrüsenhormon-Verordnungen ent- springen der Hoffnung, man könne so euthyreote Zustände erreichen. Tatsächlich führt dies aber nach einer britischen Studie in $10 \%$ der Fälle zu einer Übertherapie mit TSH-Spiegeln von 0,1 bis $0,5 \mathrm{mU} / 1$. Das bestätigt auch eine in Boston vorgestellte Untersuchung: Dort wurden 13\% der Patienten in eine klinische und weitere $16 \%$ in eine subklinische Hyperthyreose hineintherapiert, so Brabant.

Nur die Hälfte der Patienten war durch die Behandlung wirklich euthyreot. Eine Hyperthreose, auch eine subklinische, birgt aber wiederum kardiovaskuläre und Frakturrisiken. Brabant: „Wir erkaufen das eine Risiko mit der Behandlung des anderen. Da müssen wir sicherlich vorsichtig sein."

DGIM-Kongress, 2016, 9.-12. April, Mannheim 